\title{
Ex vivo mycobacterial growth inhibition assay- re-evaluation of the tool for tuberculosis immune response and vaccine efficacy
}

WHO and STOP TB have called for an ambitious goal to end TB as a global epidemic by 2035 , aiming foran average of less than 10 tuberculosis cases per 100,000 and reduce the number of deaths from TB by $95 \%{ }^{1}$. It is clear that new tools are needed to achieve this ambitious goal ${ }^{2}$. It has beenspeculated that with the optimisation of current tools, improved universal health coverage and social protection, a rate of 25 TB cases per 100,000 a year by 2035 can be achieved. However, to 'end TB' by 2035, we will need new diagnostics, drug treatments, vaccines and biomarkers.

With the need for a more effective vaccine, the continued elusiveness of a correlate of protection, there has been a resurgence of interest in mycobacterial growth inhibition assays (MGIAs), which consider a range of immune mechanisms and their complex interactions. These systems utilise whole blood or peripheral blood mononuclear cells (PBMCs), and measure the ability to inhibit growth of mycobacteria following in vitro infection.MGIA assay can also provide a unique opportunity to manipulate the host response in a very defined manner and measure the effect to control bacterial growth, e.g. by neutralisation of cytokines, depletion of specific host cell populations, or the use of genetically modified strains of both animals or mycobacteria (1).These assays are also applicable to other areas of TB research including drug evaluation and diagnostics ${ }^{3}$.

MGIA assaysmeasure the summative ability of all innate, cellmediated and humoral immune components in a sample to contribute to the control of mycobacterial growth. It can be translated between different animal species and into humans, providing a bridge between preclinical efficacy of vaccine candidates and efficacy potential in early clinical trials. Assays measuring mycobacterial growth have been used in a number of studies in recent decades, but have not proved easy to implement at scale or in vaccine trial settings $s^{4,5,6}$.

The MGIA principle is based on the principle that host cells will inhibit mycobacterial growth in cell culture ex vivo if an effective, specific immune response is induced. Bacterial numbers can be quantified using the BACTEC MGIT system, which assesses bacterial growth automatically every hour.
This system is based on fluorescence quenching by oxygen the silica gel at the bottom of the tube is non-fluorescent in the presence of the oxygen-enriched mycobacterial growth medium. Once fluorescence reaches a certain threshold as a result of bacterial growth, the tube is registered as positive, and the time to detection (TTD) is given. TTD is dependent on the initial number of bacteria used to inoculate the MGIT tube, and the relationship between initial bacterial numbers and TTD can be determined by producing a standard curve. The standard curve can then be used to convert TTD to bacterial numbers (CFU; colony forming units? ${ }^{7}$ In 2001, Wallis et al. described a whole blood MGIA to measure the bactericidal activity of anti-TB drugs. In a study of HIVnegative volunteers receiving the standard 6 month treatment for drug-sensitive pulmonary $\mathrm{TB}$, the bactericidal activity observed using MGIA, during treatment correlated with the rate of decrease of sputum bacillary load during the first 4 weeks and was associated with time to conversion to sputum negativity ${ }^{8}$. In 2013, the Wallis whole blood MGIA was employed by Fletcher et al. alongside a PBMC-based adaptation. Following primary BCG vaccination, PBMC mediated significantly enhanced growth inhibition at both 4 and 8 weeks following vaccinationwere observed ${ }^{9}$. MGIA was performed in a LTBI and helminth coinfected UK migrant cohort for the first time to observe how these subjects can control the growth of mycobacteria andwhether anthelmintic treatment has any impact on the ability of the cells to control mycobacterial growth effectively. Helminth infected patients displayed poor growth inhibition in the MGIA which was improved after anthelmintic treatment indicating this immunomodulation might be helminth mediated ${ }^{10}$.

However, this assay needs to be optimizedfor accuracy, precision, reproducibility,specificity and linearity. Some optimization of the assay has been done, for example- timing of processing the sample, BCG inoculation time, use of serum, antibiotics, BCG inoculum volume, BCG declumping methods, individual variation, role of cells and cytokines etc. Despite the numerous optimization experiments that have been performed-there is still a majorconcern about the reproducibility of this assay ${ }^{11,12}$. The earlier use of a sub- 
optimal protocol may also have resulted in the inability to detect vaccine-induced protection, for example following BCG vaccination ${ }^{13}$.

Developing biomarkers of protective immunity is difficult, in the absence of a fully understanding the host response in TB. Biomarkers of infection or of risk of progression to disease could contribute to both the development of diagnostic assays for TB and help to identify those at greater risk of progression, thus enabling smaller and less costly vaccine trials. There is an urgent need for biomarkers that can select the most promising of these TB vaccine candidates for accelerated clinical development. A rapid and immediate acceleration in bi omarker research is required in preparation for the screening of large number of new entry TB vaccine candidates in the next 5-10 years. Measuring what really matters, the ability to kill or inhibit the growth of BCG or M. tuberculosis itself, may help al so to identify useful biomarkers.

\section{REFERENCES:}

1. Zelmer AA, Tanner R, Stylianou E, Morris S, Izzo A, Williams $A$, et al. Ex vivo mycobacterial growth inhibition assay (MGIA) for tuberculosis vaccine testing- a protocol for mouse splenocytes. bioRxiv. 2015 J un 7;20560.

2. Fletcher HA, Dockrell HM. Human biomarkers: can they help us to develop a new tuberculosis vaccine? Future Microbiol. 2016 May 20;11(6):781-7.

3. Zelmer A, Tanner R, Stylianou E, Damelang T, Morris S, Izzo A, et al. A New Tool for Tuberculosis Vaccine Screening: Ex Vivo Mycobacterial Growth Inhibition Assay Indicates Bcg-Mediated Protection in a Murine Model of Tubercul osis. BMC Infect Dis. 2016;16:412.

4. Hoft DF, Worku S, Kampmann B, Whalen CC, Ellner JJ, Hirsch CS, et al. Investigation of the Relationships between Immune-Mediated Inhibition of Mycobacterial Growth and Other Potential Surrogate Markers of Protective Mycobacterium tuberculosis Immunity. J Infect Dis. 2002 Nov 15;186(10):1448-57.
5. Silver RF, Li Q, Boom WH, Ellner JJ. LymphocyteDependent Inhibition of Growth of Virulent Mycobacterium tuberculosis H37Rv Within Human Monocytes: Requirement for CD4+ T Cells in Purified Protein Derivative-Positive, But Not in Purified Protein Derivative-Negative Subjects. J Immunol. 1998 Mar 1;160(5):2408-17.

6. Worku S, Hoft DF. Differential Effects of Control and Antigen-Specific T Cells on Intracellular Mycobacterial Growth. Infect Immun. 2003 A pr;71(4):1763-73.

7. Tanner R, O'Shea MK, Fletcher HA, McShane H. In vitro mycobacterial growth inhibition assays: A tool for the assessment of protective immunity and evaluation of tuberculosis vaccine efficacy. Vaccine. 2016 Sep 7;34(39):4656-65.

8. Wallis RS, Vinhas SA, J ohnson J L, Ribeiro FC, Palaci M, Peres RL, et al. Whole Blood Bactericidal Activity during Treatment of Pulmonary Tubercul osis. J Infect Dis. 2003 Jan 15;187(2):270-8.

9. Fletcher HA, Tanner R, Wallis RS, Meyer J, Manjaly Z-R, Harris S, et al. Inhibition of Mycobacterial Growth In Vitro following Primary but Not Secondary Vaccination with Mycobacterium bovis BCG. Clin Vaccine Immunol CVI. 2013 Nov;20(11):1683-9.

10. Anwar S. Impact of helminth infection on antimycobacterial immune responses in UK migrants. [PhD Thesis] [London (UK)]: University of London; 2017

11. Levin M, Kaforou M. Predicting active tuberculosis progression by RNA analysis. The Lancet. 2016 Jun;387(10035):2268-70.

12. Tanner R, Development of Mycobacterial Growth Inhibition Assay for the early evaluation and gating of novel TB vaccine candidates. PhD [Thesis]. Oxford: University of Oxford; 2015.

13. Fletcher HA, Snowden MA, Landry B, Rida W, Satti I, Harris SA, et al. T-cell activation is an immune correlate of risk in BCG vaccinated infants. Nat Commun. 2016 Apr 12; 7:11290. $\triangle D$ Correspondence

Dr. ShahedaAnwar

Asstt. Professor

Department of Microbiology and I mmunology

BSMMU, Dhaka

Mobile: 01786-491325 